\title{
ANALYSIS OF SPLITTING METHODS FOR REACTION-DIFFUSION PROBLEMS USING STOCHASTIC CALCULUS
}

\author{
ERWAN FAOU
}

\begin{abstract}
We consider linear and nonlinear reaction-diffusion problems, and their time discretization by splitting methods. We give probabilistic interpretations of the splitting schemes, and show how these representations allow us to give error bounds for the deterministic propagator under weak hypothesis on the reaction part. To show these results, we only use the Itô formula, and basic properties of solutions of stochastic differential equations. Eventually, we show how probabilistic representations of splitting schemes can be used to derive "hybrid" numerical schemes based on Monte Carlo approximations of the splitting method itself.
\end{abstract}

\section{INTRODUCTION}

The main goal of this work is to show how probabilistic interpretations of splitting schemes for nonlinear parabolic problems can yield to deterministic estimates for the error approximation. We will also show how probabilistic representation of numerical schemes combined with Monte Carlo approximation can lead to new numerical methods that could be considered as "hybrid" Monte Carlo methods for parabolic problems.

The equations we consider are of the form

$$
\frac{\partial u}{\partial t}(t, x)=\Delta u(t, x)+g(u(t, x)), \quad u(0, x)=u_{0}(x)
$$

where $u(t, x)$ is a real function depending on the time $t \geq 0$ and the space variable $x=\left(x_{i}\right)_{i=1}^{d} \in \mathbb{R}^{d}, d \geq 1$. The operator $\Delta=\sum_{i=1}^{d} \partial_{x_{i}}^{2}$ is the Laplace operator in $\mathbb{R}^{d}$. The reaction term $u \mapsto g(u)$ is a real function defined on $\mathbb{R}$ such that $g(0)=0$. For simplicity, we often write $u(t)$ to denote the solution of (1.1) at the time $t \geq 0$. We define $e^{t \Delta} u_{0}$ and $\varphi_{t}\left(u_{0}\right)$ the solutions at the time $t \geq 0$ of the equations

$$
\partial_{t} v(t, x)=\Delta v(t, x), \quad v(0, x)=u_{0}
$$

and

$$
\partial_{t} v(t, x)=g(v(t, x)), \quad v(0, x)=u_{0}
$$

Received by the editor November 13, 2007 and, in revised form, May 13, 2008.

2000 Mathematics Subject Classification. Primary 65M15, 60H30, $65 \mathrm{C} 05$.

Key words and phrases. Splitting methods, reaction-diffusion equations, stochastic processes, Monte Carlo methods. 
respectively. In this work, we consider the approximations of solutions of (1.1) given by the Lie splitting methods:

$$
u(t) \simeq \varphi_{t}\left(e^{t \Delta} u_{0}\right)=:\left(\varphi_{t} \circ e^{t \Delta}\right)\left(u_{0}\right)
$$

and

$$
u(t) \simeq e^{t \Delta}\left(\varphi_{t}\left(u_{0}\right)\right)=:\left(e^{t \Delta} \circ \varphi_{t}\right)\left(u_{0}\right) .
$$

We will also consider the following Strang splitting method

$$
u(t) \simeq \varphi_{t / 2}\left(e^{t \Delta} \varphi_{t / 2}\left(u_{0}\right)\right)=:\left(\varphi_{t / 2} \circ e^{t \Delta} \circ \varphi_{t / 2}\right)\left(u_{0}\right) .
$$

The starting point of this work is to write the preceding approximations using the Feynman-Kac formula: For instance, we can write for all $x$,

$$
\left(e^{t \Delta} \circ \varphi_{t}\left(u_{0}\right)\right)(x)=\mathbb{E}\left(\varphi_{t}\left(u_{0}\left(X_{t}^{x}\right)\right)\right)
$$

where $X_{t}^{x}$ is the standard $d$-dimensional Wiener process in $\mathbb{R}^{d}$ (scaled by a factor $\sqrt{2}$ ) starting in $x$. A similar formula holds for the schemes (1.4) and (1.6). Using these probabilistic representations, the goal of this paper is twofold:

- Use stochastic calculus to obtain optimal bounds for the error between $u(t)$ and the previous splitting approximations. In the linear case, this can be done very easily using the Feynman-Kac formula for the exact solution itself. In the nonlinear case, it turns out that it is still possible to obtain estimates using the Itô formula. We actually do not need to have a probabilistic representation of the exact solution of (1.1), but rather only use probabilistic representations of the splitting methods themselves. At the end, we obtain deterministic bounds using stochastic methods.

- Use these probabilistic representations to derive new numerical schemes: Indeed, if $h>0$ denotes a small stepsize, we approximate $u(h)$ using (1.7) by the Monte Carlo formula

$$
u(h, x) \simeq u_{1}(x):=\frac{1}{N} \sum_{n=1}^{N} \Phi_{h}\left(u_{0}\left(X_{h ; n}^{x}\right)\right)
$$

where $\Phi_{h}$ is a numerical approximation of the flow $\varphi_{h}$ and where the $X_{h ; n}^{x}$, $n=1, \ldots, N$ are independent realizations of the process $X_{h}^{x}$. After interpolating $u_{1}$, we can iterate the algorithm and obtain a numerical scheme. This method is a compromise between fully deterministic schemes where a space approximation of the Laplace operator would be used, and Monte Carlo or particle methods where the stochastic processes are simulated up to the final time $T$. We will show by numerical experiment that schemes of the form (1.8) give good results, even for relatively small values of $N$.

The paper is organized as follows: In Section 2, we study the linear case, i.e. systems where $g(u)=V u$, with a potential function $V(x)$ that depends on the space variable $x \in \mathbb{R}^{d}$. In this situation, many results already exist: See, in particular, [10, 9, 7], the review in [13] and the reference therein. We mention in particular the results in [15] where a probabilistic method is used. As in our work, the starting point is the Feynman-Kac formula. However, the analysis is made using estimates on the probability transition kernel, while in our work we use directly the Itô formula and basic estimates of solutions of stochastic differential equations. As in [15], we obtain estimates in $L^{p}$ norms for arbitrary $p$. 
Comparing with these previous works, we only assume that $V$ is a bounded and Lipschitz function in $\mathbb{R}^{d}$. The convergence result is stated in Theorem 2.1. Its very simple proof relies on the following: We write the Feynman-Kac formula for the exact solution and for the solution of the splitting method. The difference is driven by a quadrature error of the process $V\left(X_{t}^{x}\right)$ where $V$ is the potential function and $X_{t}^{x}$ the process appearing in (1.7). We thus obtain directly the result using standard estimates for the expectation of Wiener processes.

In Section 3, we study the nonlinear case (1.1) and show the convergence of the Lie and Strang splitting methods above under smoothness assumptions on the initial conditions. The method consists of studying the stochastic process $s \mapsto U(s):=\varphi_{s}\left(u\left(t-s, X_{s}^{x}\right)\right)$. At the time $s=0$, it is equal to $u(t, x)$ and at the time $s=t$, to $\varphi_{t}\left(u_{0}\left(X_{t}\right)\right)$ whose expectation gives the splitting scheme (1.7). We use the Itô formula to expand $U(s)$, and conclude by estimating the terms in the expansion after taking the expectation. This method is familiar when working with the approximations of parabolic PDE using Monte Carlo methods; see for instance [14. The main results are given by Theorems 3.2 and 3.5 . For the analysis of splitting schemes applied to nonlinear reaction-diffusion problems using deterministic methods, we refer to 6, 3, 4. However, let us mention that the convergence results we give for splitting methods applied to nonlinear parabolic problems apparently does not exist yet in the literature.

It is worth noting that the method we use can be applied to more general situations. In particular, the results presented below extend straightforwardly to partial differential equations of the form

$$
\partial_{t} u=\operatorname{div}(A(x) \nabla u)+f(x)^{T} \nabla u+g(u)
$$

where $A(x)$ is a $d \times d$ matrix such that $A=\frac{1}{2} \sigma \sigma^{T}$ where $\sigma(x)$ is a $d \times d$ matrix, and where $f(x)$ is a $d$-dimensional vector. In this case, the stochastic process appearing in (1.7) in a splitting procedure between the linear and the nonlinear part is replaced by the solution of the stochastic differential equation

$$
\mathrm{d} X_{t}^{x}=\tilde{f}\left(X_{t}^{x}\right) \mathrm{d} t+\sigma\left(X_{t}^{x}\right) \mathrm{d} W_{t}, \quad X_{0}^{x}=x,
$$

where $\tilde{f}(x)=f(x)+\sum_{i=1}^{d} \partial_{x_{i}} A_{i j}(x)$. In particular, we never use the regularization properties of the heat equation semigroup. The other result can therefore be easily extended to the case where $A(x)$ is not positive definite. For simplicity of the presentation, we only consider the case where $A$ is the identity matrix, and $f=0$.

In Section 4 we describe a hybrid Monte Carlo method following from the representation (1.7) based on approximations of the form (1.8). We present numerical examples in the linear case, and for the Fisher-KPP and Ginzburg-Landau equations. This method is different from standard Monte Carlo or particle methods (see [1, 12]). In particular, the method differs because each stochastic process is simulated from points on a fixed grid at each time step, while in particles or classical Monte Carlo methods, the processes are simulated up to the final time. The price to pay is the interpolation made at each time-step, but the advantage is that the processes appearing in the algorithm have all small variances. This might explain why the number of realizations $N$ can seemingly be taken much smaller than usual. Let us emphasize however that a complete numerical analysis of this scheme is out of the scope of this paper. 
Let us mention that all these results also extend to the case of systems of the form

$$
\partial_{t} v=\Delta v+f(v)
$$

where $v(t, x), t \geq 0$ and $x \in \mathbb{R}^{d}$ takes values in $\mathbb{R}^{n}, n \geq 0$ and $f(v)$ a vector field in $\mathbb{R}^{n}$. In this situation, formulas like (1.7) still hold true componentwise. Such an example is given at the end of the paper by considering the Ginzburg-Landau equation.

\section{THE LINEAR CASE}

We first consider the case where the equation (1.1) is linear, that is,

$$
\partial_{t} u=\Delta u+V u, \quad u(0, x)=u_{0}(x)
$$

where $u(t, x)$ is a function of the time $t \geq 0$ and the space variable $x \in \mathbb{R}^{d}$ and where the potential $V$ is a function depending on $x \in \mathbb{R}^{d}$. In this case, we write the flow of the reaction part $\varphi_{t}\left(u_{0}\right)=e^{t V} u_{0}$.

We assume that the potential $V$ is Lipschitz. For $x \in \mathbb{R}^{d}$, let $X_{t}^{x}$ be the solution of the stochastic differential equation

$$
\mathrm{d} X_{t}^{x}=\sqrt{2} \mathrm{~d} W_{t}, \quad \text { and } \quad X_{0}=x .
$$

where $W_{t}$ is the standard $d$-dimensional Wiener process in $\mathbb{R}^{d}$ with independent components. The Feynman-Kac formula asserts that we have for all $t \geq 0$,

$$
u(t, x)=\mathbb{E}\left(u_{0}\left(X_{t}^{x}\right) \exp \left(\int_{0}^{t} V\left(X_{s}^{x}\right) \mathrm{d} s\right)\right) .
$$

Similarly, using again the Feynman-Kac formula, we have for $t \geq 0$,

$$
\left(e^{t \Delta} e^{t V} u_{0}\right)(x)=\mathbb{E}\left(u_{0}\left(X_{t}^{x}\right) e^{t V\left(X_{t}^{x}\right)}\right)
$$

and

$$
\left(e^{t V} e^{t \Delta} u_{0}\right)(x)=\mathbb{E}\left(u_{0}\left(X_{t}^{x}\right) e^{t V(x)}\right) .
$$

The goal of this section is to prove the following result:

Theorem 2.1. Assume that $V$ is bounded and Lipschitz on $\mathbb{R}^{d}$. Let $t_{0}$ be a fixed positive number. Then we have for all $p$ with $1 \leq p \leq \infty$ and for all $u_{0} \in L^{p}\left(\mathbb{R}^{d}\right)$,

$$
\left\|u(t)-\left(e^{t V} e^{t \Delta} u_{0}\right)\right\|_{L^{p}} \leq C t^{3 / 2}\left\|u_{0}\right\|_{L^{p}}
$$

and

$$
\left\|u(t)-\left(e^{t \Delta} e^{t V} u_{0}\right)\right\|_{L^{p}} \leq C t^{3 / 2}\left\|u_{0}\right\|_{L^{p}}
$$

where $u(t)$ denotes the solution of (2.1) given by (2.3) with initial value $u_{0}$ at $t=0$, and where the constants in the previous inequalities only depend on $V, t_{0}, p$ and the dimension $d$.

Proof. We have using (2.3) and (2.5),

$$
u(t, x)-\left(e^{t V} e^{t \Delta} u_{0}\right)(x)=\mathbb{E}\left(u_{0}\left(X_{t}^{x}\right)\left(e^{\int_{0}^{t} V\left(X_{s}^{x}\right) \mathrm{d} s}-e^{t V(x)}\right)\right) .
$$


For $y$ in $\mathbb{R}^{d}$, we set $\|y\|_{1}=\sum_{1 \leq i \leq d}\left|y_{i}\right|$, the $\ell^{1}$ norm in $\mathbb{R}^{d}$. Assuming that $\|V\|_{L^{\infty}} \leq$ $M_{V}$, that $V$ is Lipschitz with a constant $L_{V}$ for the norm $\|\cdot\|_{1}$, and that $|t| \leq t_{0}$ we deduce that

$$
\left|u(t, x)-\left(e^{t V} e^{t \Delta} u_{0}\right)(x)\right| \leq L_{V} e^{t_{0} M_{V}} \mathbb{E}\left(\left|u_{0}\left(X_{t}^{x}\right)\right| \int_{0}^{t}\left\|X_{s}^{x}-x\right\|_{1} \mathrm{~d} s\right) .
$$

We derive that

$$
\left\|u(t)-\left(e^{t V} e^{t \Delta} u_{0}\right)\right\|_{L^{\infty}} \leq L_{V} e^{t_{0} M_{V}}\left\|u_{0}\right\|_{L^{\infty}} \int_{0}^{t} \mathbb{E}\left\|X_{s}^{x}-x\right\|_{1} \mathrm{~d} s .
$$

But we have for all $s \in(0, t)$,

$$
\mathbb{E}\left\|X_{s}^{x}-x\right\|_{1}=\sqrt{2} \mathbb{E}\|W(s)\|_{1} \leq C s^{1 / 2},
$$

for a constant $C$ depending on $d$. This yields

$$
\left\|u(t)-\left(e^{t V} e^{t \Delta} u_{0}\right)\right\|_{L^{\infty}} \leq C t^{3 / 2}\left\|u_{0}\right\|_{L^{\infty}}
$$

where $C$ only depends on $V, t_{0}$ and $d$. This shows (2.6) in the case where $p=\infty$.

Similarly, as $X_{t}^{x}-x=\sqrt{2} W(t)$ is independent of $x$ we have for any integrable function $f$ on $\mathbb{R}^{d}$, and for any time $t \geq 0$,

$$
\int_{\mathbb{R}^{d}} f\left(X_{t}^{x}\right) \mathrm{d} x=\int_{\mathbb{R}^{d}} f(x) \mathrm{d} x, \quad \text { a.e. }
$$

Using this relation, we automatically get from (2.8) using Fubini's Theorem that

$$
\left\|u(t)-\left(e^{t V} e^{t \Delta} u_{0}\right)\right\|_{L^{1}} \leq C t^{3 / 2}\left\|u_{0}\right\|_{L^{1}}
$$

which shows (2.6) in the case where $p=1$.

For $1<p<\infty$, the equation (2.8) together with Jensen inequality for the convex function $y \mapsto y^{p}$ in the probability space $(\Omega, \mathbb{P})$ on which the stochastic process is defined imply that for all $x \in \mathbb{R}^{d}$,

$$
\left|u(t, x)-\left(e^{t V} e^{t \Delta} u_{0}\right)(x)\right|^{p} \leq L_{V} e^{t_{0} M_{V}} \mathbb{E}\left(\left|u_{0}\left(X_{t}^{x}\right)\right|^{p}\left|\int_{0}^{t}\left\|X_{s}^{x}-x\right\|_{1} \mathrm{~d} s\right|^{p}\right) .
$$

Integrating this equation in $x \in \mathbb{R}^{d}$, using Fubini's theorem and again the Jensen inequality but the for integration variable $s \in(0, t)$, we find

$$
\left\|u(t)-\left(e^{t V} e^{t \Delta} u_{0}\right)\right\|_{L^{p}}^{p} \leq C t^{p} \mathbb{E}\left(\int_{\mathbb{R}^{d}}\left|u_{0}\left(X_{t}^{x}\right)\right|^{p}\left(\frac{1}{t} \int_{0}^{t}\left\|X_{s}^{x}-x\right\|_{1}^{p} \mathrm{~d} s\right) \mathrm{d} x\right) .
$$

As $X_{s}^{x}-x=\sqrt{2} W(s)$ is independent of $x$, we have using (2.11),

$$
\left\|u(t)-\left(e^{t V} e^{t \Delta} u_{0}\right)\right\|_{L^{p}}^{p} \leq C t^{p}\left\|u_{0}\right\|_{L^{p}}^{p}\left(\frac{1}{t} \int_{0}^{t} \mathbb{E}\|W(s)\|_{1}^{p} \mathrm{~d} s\right) .
$$

The result then follows from the fact that $\mathbb{E}\|W(s)\|_{1}^{p} \leq C s^{p / 2}$ for all $s$ and for a constant depending on $p$ : This is an easy consequence of the Itô formula when $p=2 k$ for $k \in \mathbb{N}$, and for any given $p>1$, this comes from $\mathbb{E}\|W(s)\|_{1}^{p} \leq C\left(\mathbb{E}\|W(s)\|_{1}^{2 k}\right)^{\frac{p}{2 k}}$ for $k$ such that $2 k>p$.

The proof of (2.7) is similar. 
Remark 2.2. The previous $L^{\infty}$ estimates readily extend to partial differential equations of the form

$$
\partial_{t} u=\operatorname{div}(A(x) \nabla u)+f(x)^{T} \nabla u+V(x) u, \quad u(0)=u_{0}
$$

where $A(x)$ is a $d \times d$-dimensional matrix such that there exists a $d \times d$ matrix $\sigma(x)$ such that $A(x)=\frac{1}{2} \sigma(x) \sigma(x)^{T}$, and where $f(x)$ is a $d$-dimensional vector, $\nabla u$ is the gradient of $u$ in $\mathbb{R}^{d}$. In this case, the Feynman-Kac formula (2.3) is still valid, with the process $X_{t}^{x}$ satisfying the stochastic differential equation

$$
\mathrm{d} X_{t}^{x}=\tilde{f}\left(X_{t}^{x}\right) \mathrm{d} t+\sigma\left(X_{t}^{x}\right) \mathrm{d} W_{t}, \quad X_{0}^{x}=x,
$$

where $\tilde{f}(x)=f(x)+\sum_{i=1}^{d} \partial_{x_{i}}\left(\sigma(x) \sigma(x)^{T}\right)_{i j}$. In this case, the diffusion part $e^{t \Delta} u_{0}$ solution of (1.2) in the splitting scheme has to be replaced by the solution of the equation

$$
\partial_{t} u=\operatorname{div}(A(x) \nabla u)+f(x)^{T} \nabla u, \quad u(0)=u_{0},
$$

which corresponds to the stochastic differential equation (2.13). The estimates (2.6) and (2.7) for $p=\infty$ then remain valid, as long as $\sigma$ and $f$ are bounded.

Corollary 2.3. Under the assumptions of Theorem 2.1, let $\tau_{0}$ be a fixed positive number, and let $T>0$ be given. Then for all $\tau \leq \tau_{0}$ and $n$ such that $n \tau \leq T$, we have for all $p$ with $1 \leq p \leq \infty$ and all $u_{0} \in L^{p}\left(\mathbb{R}^{d}\right)$,

$$
\left\|u(n \tau)-\left(e^{\tau \Delta} e^{\tau V}\right)^{n} u_{0}\right\|_{L^{p}} \leq C \tau^{1 / 2}\left\|u_{0}\right\|_{L^{p}}
$$

and

$$
\left\|u(n \tau)-\left(e^{\tau V} e^{\tau \Delta}\right)^{n} u_{0}\right\|_{L^{p}} \leq C \tau^{1 / 2}\left\|u_{0}\right\|_{L^{p}},
$$

where $C$ only depends on $V, p, d, \tau_{0}$ and $T$.

Proof. The proof is classic, but we recall it here for completeness. It is clear that for any $p, 1 \leq p \leq \infty$, we have for all functions $u$,

$$
\left\|e^{t \Delta} u\right\|_{L^{p}} \leq\|u\|_{L^{p}} \quad \text { and } \quad\left\|e^{t V} u\right\|_{L^{p}} \leq e^{t M_{V}}\|u\|_{L^{p}}
$$

where $M_{V}=\|V\|_{L^{\infty}}$. Let $H=\Delta+V$. We have $u(t, x)=e^{t H} u_{0}$. For all functions $u$, the Duhamel formula states that

$$
e^{t H} u=e^{t \Delta} u+\int_{0}^{t} e^{(t-s) \Delta} V e^{s H} u
$$

Using Gronwall's Lemma, we deduce that any $p, 1 \leq p \leq \infty$,

$$
\left\|e^{t H} u\right\|_{L^{p}} \leq e^{t M_{V}}\|u\|_{L^{p}} .
$$

For a given $\tau$, let $S=e^{\tau H}$ and $L=e^{\tau \Delta} e^{\tau V}$. We have

$$
u(n \tau, x)-\left(e^{\tau V} e^{\tau \Delta}\right)^{n} u_{0}=S^{n} u_{0}-L^{n} u_{0}=\sum_{j=0}^{n-1} S^{n-j-1}(S-L) L^{j} u_{0} .
$$

We deduce from the previous estimates that for $p$ with $1 \leq p \leq \infty$,

$$
\left\|u(n \tau)-\left(e^{\tau V} e^{\tau \Delta}\right)^{n} u_{0}\right\|_{L^{p}} \leq \sum_{j=0}^{n-1} e^{\tau(n-j-1) M_{V}}\left\|(S-L) L^{j} u_{0}\right\|_{L^{p}} .
$$


Using (2.6), we obtain that

$$
\left\|u(n \tau)-\left(e^{\tau V} e^{\tau \Delta}\right)^{n} u_{0}\right\|_{L^{p}} \leq C \tau^{3 / 2} \sum_{j=0}^{n-1} e^{\tau(n-1) M_{V}}\left\|u_{0}\right\|_{L^{p}},
$$

provided that $\tau \leq \tau_{0}$. This yields (2.14). The second estimate is proved in a similar way.

Remark 2.4. The previous corollary shows a convergence rate in $\sqrt{\tau}$ for the Lie splitting method applied to a linear parabolic problem. This convergence rate is optimal when the splitting operator is seen as an operator from $L^{p}$ to itself. To obtain a better convergence rate, we have to assume more regularity for the potential function and for the initial value. For instance, in the case where $V$ and $u_{0}$ are $C^{1}$ with bounded derivative, we recover the local order 2 , but with a constant depending on the derivatives of $u_{0}$ and $V$.

Similarly, with the assumptions of Theorem 2.1, no better estimate can be obtained for the Strang splitting scheme $e^{t V / 2} e^{t \Delta} e^{t V / 2}$. To obtain a better estimate, we have to assume that $V$ is more regular; see [15, 10.

\section{THE NONLINEAR CASE}

Let us now consider the equation

$$
\partial_{t} u=\Delta u+g(u), \quad u(0, x)=u_{0}(x)
$$

where $g(u)$ is a function depending on $u$. We assume that $g \in C^{2}(\mathbb{R}, \mathbb{R})$ and that there exist constants $M_{i}, i=1,2$ such that for all $u \in \mathbb{R}$, we have

$$
\left|g^{\prime}(u)\right| \leq M_{1}, \quad\left|g^{\prime \prime}(u)\right| \leq M_{2} .
$$

We will moreover assume that $g(0)=0$.

To avoid technical details, we will only deal with $L^{\infty}$ estimates in this section. For a given function $v(x), x \in \mathbb{R}^{d}$, we denote by $\nabla u$ the associated column gradient vector and $\nabla^{2} u$ the Hessian matrix. When the following norms make sense, we set

$$
\|\nabla u\|_{L^{\infty}}:=\max _{i=1}^{d} \sup _{x \in \mathbb{R}^{d}}\left|\frac{\partial v}{\partial x_{i}}(x)\right|
$$

and

$$
\left\|\nabla^{2} u\right\|_{L^{\infty}}:=\max _{i, j=1}^{d} \sup _{x \in \mathbb{R}^{d}}\left|\frac{\partial^{2} v}{\partial x_{i} \partial x_{j}}(x)\right| .
$$

Under the assumptions (3.2), it is clear from the Duhamel formula that

$$
u(t, x)=e^{t \Delta} u_{0}+\int_{0}^{t} e^{(t-s) \Delta} g(u(s, x)) \mathrm{d} s
$$

and from classical estimates for the heat kernel, that the following estimates hold: For all $t \geq 0$, if $u(t)$ denotes the solution of (3.1) at time $t$, we have

$$
\|u(t)\|_{L^{\infty}} \leq e^{t M_{1}}\left\|u_{0}\right\|_{L^{\infty}}, \quad\|\nabla u(t)\|_{L^{\infty}} \leq e^{t M_{1}}\left\|\nabla u_{0}\right\|_{L^{\infty}}
$$

and

$$
\left\|\nabla^{2} u(t)\right\|_{L^{\infty}} \leq e^{t M_{1}}\left(\left\|\nabla^{2} u_{0}\right\|_{L^{\infty}}+t M_{2} e^{2 t M_{1}}\left\|\nabla u_{0}\right\|_{L^{\infty}}^{2}\right) .
$$

Let $\varphi_{t}(u)$ be the flow associated with the differential equation

$$
\forall u \in \mathbb{R}, \quad \frac{\mathrm{d}}{\mathrm{d} t} \varphi_{t}(u)=g\left(\varphi_{t}(u)\right), \quad \text { and } \quad \varphi_{0}(u)=u .
$$


We will denote by $\varphi_{t}^{\prime}(u)$ and $\varphi_{t}^{\prime \prime}(u)$ the derivatives of the flow with respect to the initial condition, solutions of the equations

$$
\frac{\mathrm{d}}{\mathrm{d} t} \varphi_{t}^{\prime}(u)=g^{\prime}\left(\varphi_{t}(u)\right) \varphi_{t}^{\prime}(u), \quad \text { with } \quad \varphi_{0}^{\prime}(u)=1
$$

and

$$
\frac{\mathrm{d}}{\mathrm{d} t} \varphi_{t}^{\prime \prime}(u)=g^{\prime \prime}\left(\varphi_{t}(u)\right) \varphi_{t}^{\prime}(u)^{2}+g^{\prime}\left(\varphi_{t}(u)\right) \varphi_{t}^{\prime \prime}(u), \quad \text { with } \quad \varphi_{0}^{\prime \prime}(u)=0 .
$$

We deduce immediately from the preceding equations that

$$
\forall u \in \mathbb{R}, \quad \forall t \geq 0, \quad\left|\varphi_{t}^{\prime}(u)\right| \leq e^{t M_{1}} \quad \text { and }\left|\varphi_{t}^{\prime \prime}(u)\right| \leq t M_{2} e^{3 t M_{1}} .
$$

Lemma 3.1. With the preceding notations, we have for all $v \in \mathbb{R}$ and all $s \geq 0$,

$$
\varphi_{s}^{\prime}(v) g(v)=g\left(\varphi_{s}(v)\right)
$$

Proof. Let

$$
Y(s, v)=\varphi_{s}^{\prime}(v) g(v)-g\left(\varphi_{s}(v)\right) .
$$

We have that for all $v, Y(0, v)=0$. Now we compute easily that for a fixed $v$,

$$
\begin{aligned}
\frac{\mathrm{d}}{\mathrm{d} s} Y(s, v) & =g^{\prime}\left(\varphi_{s}(v)\right) \varphi_{s}^{\prime}(v) g(v)-g^{\prime}\left(\varphi_{s}(v)\right) g\left(\varphi_{s}(v)\right), \\
& =g^{\prime}\left(\varphi_{s}(v)\right) Y(s, v),
\end{aligned}
$$

and this shows that for all $s \geq 0, Y(s, v)=0$.

3.1. Lie splitting. Let us first consider the Lie splitting methods:

$$
u(t) \simeq\left(e^{t \Delta} \circ \varphi_{t}\right)\left(u_{0}\right) \text { and } u(t) \simeq\left(\varphi_{t} \circ e^{t \Delta}\right)\left(u_{0}\right) .
$$

The Feynman-Kac representations of these splitting methods can be written as

$$
e^{t \Delta}\left(\varphi_{t}\left(u_{0}(x)\right)\right)=\mathbb{E}\left(\varphi_{t}\left(u_{0}\left(X_{t}^{x}\right)\right)\right)
$$

and

$$
\left.\varphi_{t}\left(e^{t \Delta} u_{0}(x)\right)\right)=\varphi_{t}\left(\mathbb{E}\left(u_{0}\left(X_{t}^{x}\right)\right)\right)
$$

where $X_{t}^{x}$ is the stochastic process defined in (2.2).

The following result gives a local estimate in $L^{\infty}$ norm for the difference between the exact solution of (3.1) and its approximation by the Lie splitting methods. We assume here that we can compute exactly the solution of the splitting methods. Notice that such a result does not seem to exist in the literature yet, even using stronger regularity assumptions and deterministic methods.

Theorem 3.2. Assume that $g \in C^{2}(\mathbb{R})$ satisfies the hypothesis $(3.2)$ and $g(0)=0$. Let $t_{0}$ be a fixed positive number. Assume that $\nabla u_{0}(x) \in L^{\infty}\left(\mathbb{R}^{d}\right)$, Then we have for all $t \leq t_{0}$,

$$
\left\|u(t)-\left(e^{t \Delta} \circ \varphi_{t}\right)\left(u_{0}\right)\right\|_{L^{\infty}} \leq C t^{2}\left\|\nabla u_{0}\right\|_{L^{\infty}}^{2}
$$

and

$$
\left\|u(t)-\left(\varphi_{t} \circ e^{t \Delta}\right)\left(u_{0}\right)\right\|_{L^{\infty}} \leq C t^{2}\left\|\nabla u_{0}\right\|_{L^{\infty}}^{2}
$$

where $u(t)$ denote the solution of (3.1) with initial value $u_{0}$ at $t=0$, and where the constants in the previous inequalities only depend on $t_{0}, g$ and the dimension $d$. 
Proof. Let $R(t, x)=u(t, x)-e^{t \Delta}\left(\varphi_{t}\left(u_{0}(x)\right)\right)$. We define for $s \in(0, t)$,

$$
U(s)=\varphi_{s}\left(u\left(t-s, X_{s}^{x}\right)\right) .
$$

We have $U(0)=u(t, x)$ and $U(t)=\varphi_{t}\left(u_{0}\left(X_{t}\right)\right)$ so that

$$
R(t, x)=-\mathbb{E} \int_{0}^{t} \mathrm{~d} U(s) .
$$

Now we compute using the Itô formula that

$$
\begin{aligned}
\mathrm{d} u\left(t-s, X_{s}^{x}\right)= & -\partial_{t} u\left(t-s, X_{s}^{x}\right) \mathrm{d} s \\
& +\sqrt{2} \nabla u\left(t-s, X_{s}^{x}\right)^{T} \mathrm{~d} W_{s}+\Delta u\left(t-s, X_{s}^{x}\right) \mathrm{d} s
\end{aligned}
$$

and thus using (3.1)

$$
\mathrm{d} u\left(t-s, X_{s}^{x}\right)=-g\left(u\left(t-s, X_{s}^{x}\right)\right) \mathrm{d} s+\sqrt{2} \nabla u\left(t-s, X_{s}^{x}\right)^{T} \mathrm{~d} W_{s} .
$$

Using again the Itô formula, we obtain

$$
\begin{aligned}
\mathrm{d} U(s)= & \varphi_{s}^{\prime}\left(u\left(t-s, X_{s}\right)\right)\left(-g\left(u\left(t-s, X_{s}^{x}\right)\right) \mathrm{d} s+\sqrt{2} \nabla u\left(t-s, X_{s}^{x}\right)^{T} \mathrm{~d} W_{s}\right) \\
& +g\left(\varphi_{s}\left(u\left(t-s, X_{s}\right)\right)\right) \mathrm{d} s+\varphi_{s}^{\prime \prime}\left(u\left(t-s, X_{s}\right)\right)\left\|\nabla u\left(t-s, X_{s}^{x}\right)\right\|_{2}^{2} \mathrm{~d} s,
\end{aligned}
$$

where $\|\cdot\|_{2}$ denotes the Euclidean norm in $\mathbb{R}^{d}$.

We deduce from (3.13), Lemma 3.1 and the martingale property of the Itô integral, that we have

$$
R(t, x)=\int_{0}^{t} \mathbb{E} \varphi_{s}^{\prime \prime}\left(u\left(t-s, X_{s}^{x}\right)\right)\left\|\nabla u\left(t-s, X_{s}^{x}\right)\right\|_{2}^{2} \mathrm{~d} s .
$$

Using (3.4) and (3.9), we find that for $t \leq t_{0}$,

$$
\|R(t, x)\|_{L^{\infty}} \leq C t^{2}\left\|\nabla u_{0}\right\|_{L^{\infty}}^{2}
$$

where $C$ only depends on $t_{0}, g$ and the dimension $d$. This shows (3.10).

Now we have that

$$
\begin{aligned}
\varphi_{t}\left(u_{0}\left(X_{t}^{x}\right)\right) & -\varphi_{t}\left(\mathbb{E}\left(u_{0}\left(X_{t}^{x}\right)\right)\right)=\left(u_{0}\left(X_{t}^{x}\right)-\mathbb{E} u_{0}\left(X_{t}^{x}\right)\right) \varphi_{t}^{\prime}\left(\mathbb{E} u_{0}\left(X_{t}^{x}\right)\right) \\
& \left.+\frac{1}{2}\left(u_{0}\left(X_{t}^{x}\right)-\mathbb{E} u_{0}\left(X_{t}^{x}\right)\right)^{2} \int_{0}^{1} \varphi_{t}^{\prime \prime}\left((1-\sigma) \mathbb{E} u_{0}\left(X_{t}^{x}\right)\right)+\sigma u_{0}\left(X_{t}^{x}\right)\right) \mathrm{d} \sigma .
\end{aligned}
$$

We deduce using (3.9) that there exists a constant $C$ such that

$$
\left|\mathbb{E} \varphi_{t}\left(u_{0}\left(X_{t}^{x}\right)\right)-\varphi_{t}\left(\mathbb{E}\left(u_{0}\left(X_{t}^{x}\right)\right)\right)\right| \leq C t \mathbb{E}\left|u_{0}\left(X_{t}^{x}\right)-\mathbb{E} u_{0}\left(X_{t}^{x}\right)\right|^{2} .
$$

But we have

$$
u_{0}\left(X_{t}^{x}\right)=u_{0}(x)+\left(X_{t}^{x}-x\right)^{T} \int_{0}^{1} \nabla u_{0}\left((1-\sigma) x+\sigma X_{t}^{x}\right) \mathrm{d} \sigma .
$$

Hence it is clear that

$$
\mathbb{E}\left|u_{0}\left(X_{t}^{x}\right)-\mathbb{E} u_{0}\left(X_{t}^{x}\right)\right|^{2} \leq t\left\|\nabla u_{0}\right\|_{L^{\infty}}^{2} .
$$

This yields

$$
\begin{aligned}
& \left\|\left(e^{t \Delta} \circ \varphi_{t}\right)\left(u_{0}\right)-\left(\varphi_{t} \circ e^{t \Delta}\right)\left(u_{0}\right)\right\|_{L^{\infty}} \\
& \quad=\left\|\mathbb{E} \varphi_{t}\left(u_{0}\left(X_{t}^{x}\right)\right)-\varphi_{t}\left(\mathbb{E}\left(u_{0}\left(X_{t}^{x}\right)\right)\right)\right\|_{L^{\infty}} \\
& \quad \leq C t^{2}\left\|\nabla u_{0}\right\|_{L^{\infty}}^{2} .
\end{aligned}
$$


This estimate, combined with (3.10), then yields (3.11).

The next result gives the global result following from the previous theorem:

Corollary 3.3. Under the assumptions of Theorem 2.1, let $\tau_{0}$ be a fixed positive number, and let $T>0$ be given. For all $\tau \in\left(0, \tau_{0}\right)$, let $u_{k}$ be the sequence of functions defined recursively by $u_{k}=\left(e^{\tau \Delta} \circ \varphi_{\tau}\right)\left(u_{k-1}\right), k \geq 1$. Then for all $n$ such that $n \tau \leq T$, we have

$$
\left\|u(n \tau)-u_{n}\right\|_{L^{\infty}} \leq C \tau\left\|\nabla u_{0}\right\|_{L^{\infty}}^{2},
$$

where $C$ only depends on $g, d, \tau_{0}$ and $T$. The same result holds for the sequence of functions defined by the propagator $\varphi_{\tau} \circ e^{\tau \Delta}$.

Proof. For a given function $u_{0}$, we define the function $\Phi\left(t ; u_{0}\right)$ as the solution of (3.1) at time $t$. It is clear that we have

$$
\forall t \geq 0, \quad \forall s \geq 0, \quad \Phi\left(t+s ; u_{0}\right)=\Phi\left(t ; \Phi\left(s ; u_{0}\right)\right) .
$$

Moreover, using the Duhamel formula (3.3), we have for all $t \geq 0$ and all functions $u_{0}$ and $v_{0}$ in $L^{\infty}\left(\mathbb{R}^{d}\right)$,

$$
\left\|\Phi\left(t ; u_{0}\right)-\Phi\left(t ; v_{0}\right)\right\|_{L^{\infty}} \leq e^{t M_{1}}\left\|u_{0}-v_{0}\right\|_{L^{\infty}} .
$$

Let $\Psi_{\tau}=e^{\tau \Delta} \circ \varphi_{\tau}$. We can write $u_{n}=\Psi_{\tau}^{n}\left(u_{0}\right)$. Now we have

$$
\Phi\left(n \tau ; u_{0}\right)-\Psi_{\tau}^{n}\left(u_{0}\right)=\sum_{j=0}^{n-1} \Phi\left((n-j) \tau ; \Psi_{\tau}^{j}\left(u_{0}\right)\right)-\Phi\left((n-j-1) \tau ; \Psi_{\tau}^{j+1}\left(u_{0}\right)\right)
$$

and hence

$$
\left\|u(n \tau)-u_{n}\right\|_{L^{\infty}} \leq \sum_{j=0}^{n-1} e^{(n-j-1) \tau M_{1}}\left\|\Phi\left(\tau ; \Psi_{\tau}^{j}\left(u_{0}\right)\right)-\Psi_{\tau}^{j+1}\left(u_{0}\right)\right\|_{L^{\infty}} .
$$

Using (3.11) we get

$$
\left\|u(n \tau)-u_{n}\right\|_{L^{\infty}} \leq \tau^{2} \sum_{j=0}^{n-1} e^{(n-j-1) \tau M_{1}}\left\|\nabla \Psi_{\tau}^{j}\left(u_{0}\right)\right\|_{L^{\infty}}^{2} .
$$

Now for a given function $v$, we have

$$
\nabla \Psi_{\tau}(v)=e^{\tau \Delta} \varphi_{\tau}^{\prime}(v) \nabla v
$$

whence using (3.9),

We deduce that

$$
\left\|\nabla \Psi_{\tau}(v)\right\|_{L^{\infty}} \leq e^{\tau M_{1}}\|\nabla v\|_{L^{\infty}} .
$$

$$
\left\|\nabla \Psi_{\tau}^{j}\left(u_{0}\right)\right\|_{L^{\infty}} \leq e^{\tau j M_{1}}\left\|\nabla u_{0}\right\|_{L^{\infty}} .
$$

Hence using (3.16),

$$
\left\|u(n \tau)-u_{n}\right\|_{L^{\infty}} \leq \tau^{2} \sum_{j=0}^{n-1} e^{(n-j-1) \tau M_{1}+2 j \tau M_{1}}\left\|\nabla u_{0}\right\|_{L^{\infty}}^{2}
$$

and this yields the result with $C \leq e^{3 T M_{1}}$. 
3.2. Strang splitting. We now study the approximation given by the Strang splitting $\varphi_{t / 2} \circ e^{t \Delta} \circ \varphi_{t / 2}$. We make the assumptions that $g$ is a $C^{3}$ function on $\mathbb{R}$ satisfying $g(0)=0$. We also assume that there exist constants $M_{i}, i=0,1,2,3$ such that for all $u \in \mathbb{R}$, we have

$$
|g(u)| \leq M_{0}, \quad\left|g^{\prime}(u)\right| \leq M_{1}, \quad\left|g^{\prime \prime}(u)\right| \leq M_{2} \quad \text { and } \quad\left|g^{\prime \prime \prime}(u)\right| \leq M_{3} .
$$

Under these assumptions, we can prove the following result concerning the asymptotic expansion of the derivative of the flow $\varphi_{t}$ associated with $g$ :

Lemma 3.4. Assume that $g \in C^{3}(\mathbb{R})$ satisfies (3.17) and $g(0)=0$. Let $\varphi_{t}(u)$ be the flow defined in (3.6). Then for a given $t_{0}>0$, there exist a constant $C$ depending on $M_{i}, i=0, \ldots, 3$ such that for all $u \in \mathbb{R}$ and all $t<t_{0}$, we have

$$
\forall u \in \mathbb{R}, \quad\left|\varphi_{t}^{\prime \prime}(u)-t g^{\prime \prime}(u)\right| \leq C t^{2} .
$$

Proof. From (3.7) and (3.9) we easily get that

$$
\forall u \in \mathbb{R}, \quad\left|\varphi_{t}^{\prime}(u)-1\right| \leq t M_{1} e^{t M_{1}} .
$$

Moreover, under the assumption (3.17), we have that for all $u \in \mathbb{R}$ and all $t \geq 0$,

$$
\left|\varphi_{t}(u)-u\right| \leq t M_{0}
$$

To prove (3.18), we set $V(t)=\varphi_{t}^{\prime \prime}(u)-t g^{\prime \prime}(u)$. We have

$$
\frac{\mathrm{d}}{\mathrm{d} t} V(t)=g^{\prime \prime}\left(\varphi_{t}(u)\right) \varphi_{t}^{\prime}(u)^{2}-g^{\prime \prime}(u)+g^{\prime}\left(\varphi_{t}(u)\right) \varphi_{t}^{\prime \prime}(u), \quad \text { with } \quad V(0)=0 .
$$

We can rewrite the previous equation as

$$
\frac{\mathrm{d}}{\mathrm{d} t} V(t)=\left(g^{\prime \prime}\left(\varphi_{t}(u)\right)-g^{\prime \prime}(u)\right) \varphi_{t}^{\prime}(u)^{2}+g^{\prime \prime}(u)\left(\varphi_{t}^{\prime}(u)^{2}-1\right)+g^{\prime}\left(\varphi_{t}(u)\right) \varphi_{t}^{\prime \prime}(u)
$$

and this yields, using (3.9),

$$
\left|\frac{\mathrm{d}}{\mathrm{d} t} V(t)\right| \leq t e^{2 t M_{1}} M_{3} M_{0}+t M_{2} M_{1} e^{t M_{1}}\left(1+e^{t M_{1}}\right)+t M_{1} M_{2} e^{3 t M_{1}} .
$$

This gives (3.18) after integrating from 0 to $t<t_{0}$.

Theorem 3.5. Assume that $g \in C^{3}(\mathbb{R})$ satisfies (3.17) and $g(0)=0$. Let $t_{0}$ be a fixed positive number. Assume that $\nabla u_{0}$ and $\nabla^{2} u_{0}$ are in $L^{\infty}\left(\mathbb{R}^{d}\right)$, then we have for all $t \leq t_{0}$,

$$
\begin{aligned}
& \left\|u(t)-\left(\varphi_{t / 2} \circ e^{t \Delta} \circ \varphi_{t / 2}\right)\left(u_{0}\right)\right\|_{L^{\infty}} \\
& \quad \leq C t^{5 / 2}\left\|\nabla u_{0}\right\|_{L^{\infty}}\left(\left\|\nabla^{2} u_{0}\right\|_{L^{\infty}}+\left\|\nabla u_{0}\right\|_{L^{\infty}}^{2}+t^{1 / 2}\left\|\nabla u_{0}\right\|_{L^{\infty}}\right)
\end{aligned}
$$

where $u(t)$ denotes the solution of (3.1) with initial value $u_{0}$ at $t=0$, and where the constants in the previous inequalities only depend on $t_{0}, g$ and the dimension $d$.

Proof. We consider now $U(s)=\varphi_{s-t / 2}\left(u\left(t-s, X_{s}^{x}\right)\right)$. We have $U(0)=\varphi_{-t / 2}(u(t, x))$ and $U(t)=\varphi_{t / 2}\left(u_{0}\left(X_{t}\right)\right)$. We set $R(t, x)=U(0)-U(t)$. The same procedure as in the proof of Theorem 3.2 shows that (compare with (3.14))

$$
R(t, x)=\int_{0}^{t} \mathbb{E} \varphi_{s-t / 2}^{\prime \prime}\left(u\left(t-s, X_{s}^{x}\right)\right)\left\|\nabla u\left(t-s, X_{s}^{x}\right)\right\|_{2}^{2} \mathrm{~d} s .
$$


Using (3.18), we see that

$$
R(t, x)=R_{1}(t, x)+R_{2}(t, x)
$$

where

$$
R_{1}(t, x)=\int_{0}^{t}\left(s-\frac{t}{2}\right) \mathbb{E} g^{\prime \prime}\left(u\left(t-s, X_{s}^{x}\right)\right)\left\|\nabla u\left(t-s, X_{s}^{x}\right)\right\|_{2}^{2} \mathrm{~d} s,
$$

and $\left\|R_{2}(t, x)\right\|_{L^{\infty}} \leq C t^{3}\left\|\nabla u_{0}\right\|_{L^{\infty}}^{2}$ for a constant $C$ depending on $g$ and $t_{0}$.

Let $f(s)=\mathbb{E} g^{\prime \prime}\left(u\left(t-s, X_{s}^{x}\right)\right)\left\|\nabla u\left(t-s, X_{s}^{x}\right)\right\|_{2}^{2}$. We have that

$$
\begin{aligned}
R_{1}(t, x) & =\int_{0}^{t}\left(s-\frac{t}{2}\right) f(s) \mathrm{d} s \\
& =\int_{0}^{t / 2} s\left(f\left(\frac{t}{2}+s\right)-f\left(\frac{t}{2}-s\right)\right) \mathrm{d} s .
\end{aligned}
$$

Let $v(s)=u\left(t-s, X_{s}^{x}\right)$ for $s \in(0, t)$. We know from (3.12) that

$$
\mathrm{d} v(s)=-g(v(s)) \mathrm{d} s+\sqrt{2} \nabla v(s)^{T} \mathrm{~d} W_{s},
$$

and (3.4) shows that for all $s \leq t_{0}$,

$$
\|\nabla v(s)\|_{L^{\infty}} \leq e^{t_{0} M_{1}}\left\|\nabla u_{0}\right\|_{L^{\infty}} .
$$

By definition, we have that

$$
f(s)=\mathbb{E} g^{\prime \prime}(v(s))\|\nabla v(s)\|_{2}^{2} .
$$

Hence, we have

$$
\begin{aligned}
f\left(\frac{t}{2}+s\right)-f\left(\frac{t}{2}-s\right) & =\mathbb{E}\|\nabla v(t / 2+s)\|_{2}^{2}\left(g^{\prime \prime}(v(t / 2+s))-g^{\prime \prime}(v(t / 2-s))\right) \\
& +\mathbb{E} g^{\prime \prime}(v(t / 2-s))\left(\|\nabla v(t / 2+s)\|_{2}^{2}-\|\nabla v(t / 2-s)\|_{2}^{2}\right) .
\end{aligned}
$$

We deduce from this relation and from (3.22) that for $s \in(0, t / 2)$,

$$
\begin{array}{r}
\left|f\left(\frac{t}{2}+s\right)-f\left(\frac{t}{2}-s\right)\right| \leq M_{3} e^{t_{0} M_{1}}\left\|\nabla u_{0}\right\|_{L^{\infty}}^{2} \mathbb{E}|v(t / 2+s)-v(t / 2-s)| \\
+2 M_{2} e^{t_{0} M_{1}}\left\|\nabla u_{0}\right\|_{L^{\infty}} \mathbb{E}\|\nabla v(t / 2+s)-\nabla v(t / 2-s)\|_{2} .
\end{array}
$$

Using (3.21), we see that there exists a constant $C$ only depending on $g, t_{0}$ and $d$ such that the first term in the right-hand side can be bounded by

$$
C\left\|\nabla u_{0}\right\|_{L^{\infty}}^{2}\left(t+\sqrt{t}\left\|\nabla u_{0}\right\|_{L^{\infty}}\right) .
$$

To bound the second term, we see that

$$
\mathrm{d} \nabla v(s)=-g^{\prime}(v(s)) \nabla v(s) \mathrm{d} s+\sqrt{2} \nabla^{2} v(s) \mathrm{d} W_{s},
$$

and hence, using (3.5), the second term in the right-hand side of (3.23) is bounded by

$$
C\left\|\nabla u_{0}\right\|_{L^{\infty}}\left(t\left\|\nabla u_{0}\right\|_{L^{\infty}}+\sqrt{t}\left\|\nabla^{2} u_{0}\right\|_{L^{\infty}}+t^{3 / 2}\left\|\nabla u_{0}\right\|_{L^{\infty}}^{2}\right) .
$$

for a constant $C$ only depending on $g, t_{0}$ and $d$. The previous estimates and the equation (3.20) yield that $\|R(t, x)\|_{L^{\infty}}$ is bounded by the right-hand side of (3.19). We conclude using the fact that

$$
u(t, x)-\left(\varphi_{t / 2} \circ e^{t \Delta} \circ \varphi_{t / 2}\right)\left(u_{0}\right)=\varphi_{t / 2}(U(0))-\varphi_{t / 2}(U(t))
$$


so that using (3.9)

$$
\left|u(t, x)-\left(\varphi_{t / 2} \circ e^{t \Delta} \circ \varphi_{t / 2}\right)\left(u_{0}\right)\right| \leq e^{t M_{1}}|R(t, x)|
$$

and this yields the result.

Corollary 3.6. Under the assumptions of Theorem 2.1, let $\tau_{0}$ be a fixed positive number, and let $T>0$ be given. For all $\tau \in\left(0, \tau_{0}\right)$, let $u_{k}$ be the sequence of functions defined recursively by $u_{k}=\left(\varphi_{\tau / 2} \circ e^{\tau \Delta} \circ \varphi_{\tau / 2}\right)\left(u_{k-1}\right), k \geq 1$. Let $n$ be an integer such that $n \tau \leq T$, then we have

$$
\begin{aligned}
& \left\|u(n \tau)-u_{n}\right\|_{L^{\infty}} \\
& \quad \leq C \tau^{3 / 2}\left\|\nabla u_{0}\right\|_{L^{\infty}}\left(\left\|\nabla^{2} u_{0}\right\|_{L^{\infty}}+\left\|\nabla u_{0}\right\|_{L^{\infty}}^{2}+\tau^{1 / 2}\left\|\nabla u_{0}\right\|_{L^{\infty}}\right)
\end{aligned}
$$

where $C$ only depends on $g, d, \tau_{0}$ and $T$.

The proof of this result is essentially the same as the proof of Corollary 3.3 by using Theorem 3.5 .

Remark 3.7. As mentioned in the introduction and in Remark 2.2, the previous results extend to partial differential equations of the form (1.9), under regularity assumptions on $\sigma(x)$ and $f(x)$.

\section{A hybrid Monte Carlo algorithm}

We now define new algorithms based on formulas of the form (1.7) for approximating the equation (1.1).

Let $\delta x$ be a given positive number. For a multi-index $i=\left(i_{1}, \ldots, i_{d}\right)$, let $x_{i}$ be the points $i(\delta x), i \in[-M, M]^{d}$ defining a grid in $\mathbb{R}^{d}(M \in \mathbb{N})$. For simplicity, we consider here uniform grid points, but the principle extends easily to more general situations.

Let $h>0$ be a stepsize, and let $\Phi_{h}$ be a numerical scheme approaching the exact flow $\varphi_{t}$ at the time $t=h$. Note that in the linear case, we can take $\Phi_{h}\left(u_{0}(x)\right)=$ $e^{h V(x)} u_{0}(x)$ so that there is no numerical approximation for the reaction part.

Let $u_{k}^{i}$ be approximations of the solution $u\left(t_{k}, x_{i}\right)$ at the time $t_{k}=k h, k \in \mathbb{N}$ and at the grid points $x_{i}$. The following scheme yields an approximation of the splitting method (1.7): For $i \in[-M, M]^{d}$, we define

$$
u_{k+1}^{i}=\frac{1}{N} \sum_{n=1}^{N} \Phi_{h}\left(\mathcal{I} u_{k}\left(X_{h ; n}^{x_{i}}\right)\right)
$$

where $X_{h ; n}^{x_{i}}, n=1, \ldots, N$, are independent realizations of the Wiener process $X_{h}^{x_{i}}$. Here $\mathcal{I} u_{k}(x)$ is an interpolation function of the values $u_{k}^{j}$ at the grid points $x_{j}$. Note that $\mathcal{I} u_{k}$ can be defined in different ways using linear interpolation, B-splines or finite elements.

In the more general case (1.9), a numerical discretization of the process (1.10) has to be used to simulate the $X_{h ; n}^{x_{i}}$.

Similarly, a discretization of the splitting scheme

$$
\left(\left(\varphi_{t} \circ e^{t \Delta}\right)\left(u_{0}\right)\right)(x)=\varphi_{t}\left(\mathbb{E} u_{0}\left(X_{t}^{x}\right)\right)
$$


can be written

$$
u_{k+1}^{i}=\Phi_{h}\left(\frac{1}{N} \sum_{n=1}^{N} \mathcal{I} u_{k}\left(X_{h ; n}^{x_{i}}\right)\right)
$$

and the discretization of the Strang splitting (1.6) can be written

$$
u_{k+1}^{i}=\Phi_{h / 2}\left(\frac{1}{N} \sum_{n=1}^{N} \Phi_{h / 2}\left(\mathcal{I} u_{k}\left(X_{h ; n}^{x_{i}}\right)\right)\right) .
$$

It is not the goal of this paper to rigourously study the convergence properties of these algorithms. In particular, they may heavily depend on the tackled problem and choice of the spatial discretization and interpolation procedure. Let us stress, however, that in the case of regular grids, each interpolation step will produce a local error of the form $(\delta x)^{r}$ where $r$ is the order of the interpolant and $\delta x$ a characteristic space discretization parameter. Typically, this error will accumulate in time to yield a global error of order $(\delta x)^{r} / h$ for a fixed time of simulation. Hence, we believe that a convergence result relies on an anti-CFL condition which is less restrictive for a better interpolant. Concerning the analysis of the statistical error produced by the Monte Carlo discretization, it is clearly out of the scope of this paper.

In the next three subsections, we give numerical examples and point out some particular features of these schemes.

4.1. A linear example. We first consider the case of the linear equation

$$
\partial_{t} u(t, x)=\frac{1}{2} \Delta u(t, x)-\frac{1}{2} x^{2} u(t, x),
$$

in the case where $d=1$. The solution of this equation can be computed explicitly. We take the initial condition $u_{0}(x)=\exp \left(-x^{2} / 2\right)$, so that we have $u(t, x)=\exp \left(-\left(x^{2}+t\right) / 2\right)$. We use the splitting method (4.2) with linear interpolation. As the problem is linear, the reaction part is computed exactly (i.e. we take $\left.\Phi_{h}(u)=\exp \left(-\frac{h}{2} x^{2}\right) u\right)$.

In Figure 1, we plot the exact solution and the numerical solution computed with the previous algorithm: First with the "rough" parameters $h=0.1, \delta x=0.1$, $N=10$ (up) and then with the more refined parameters $h=0.02, \delta x=0.05$ and $N=50$ (down). We plot the results at times $t=1,2$ and 3 . Note that even for relative small value of the Monte Carlo parameter $N$, the results are not bad.

4.2. Fisher-KPP equation. We consider now the Fisher-KPP equation in $\mathbb{R}$ (see [8, 11]):

$$
\partial_{t} u=\Delta u+u-u^{2} \quad \text { with } \quad u(0, x)=u_{0}(x) .
$$

We consider the initial condition given by

$$
u_{0}(x)=\left\{\begin{array}{lll}
1 & \text { if } & x \leq 0 \\
0 & \text { if } & x>0
\end{array}\right.
$$

We use the scheme (4.2) with linear interpolation. The numerical flow $\Phi_{h}$ is the classical Euler scheme.

In Figure 2, we plot the propagating front at each integer times $t \in \mathbb{N}$ first with $h=0.1, \delta x=0.1, N=10$ (left) and then $h=0.02, \delta x=0.05$ and $N=50$ (right). 

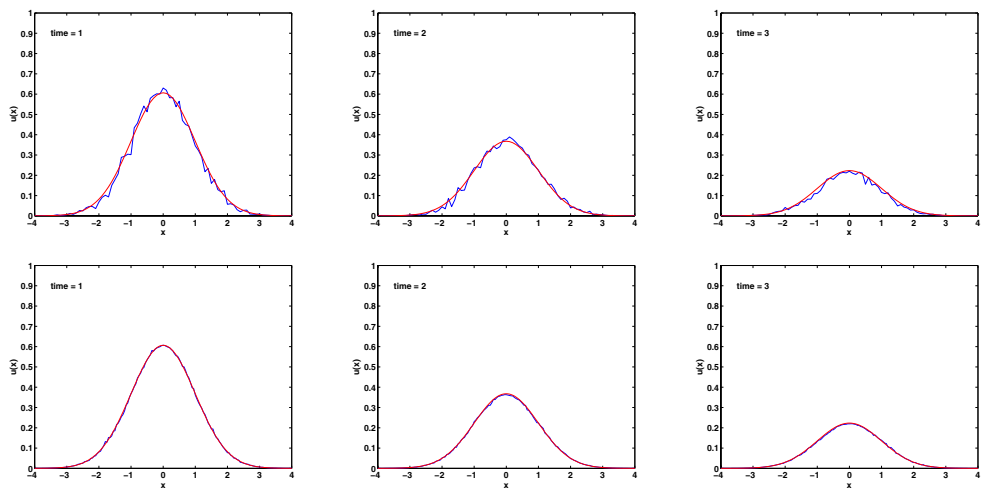

Figure 1. Solutions of the linear problem with $h=0.1, \delta x=0.1$ and $N=10$ (up) $h=0.02, \delta x=0.05$ and $N=50$ (down).
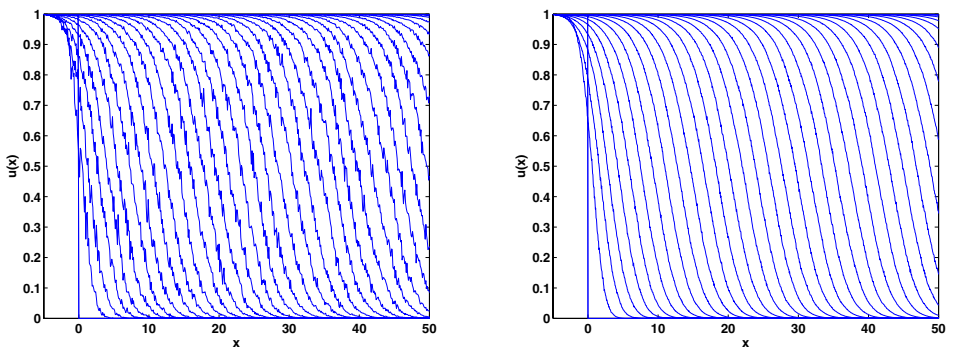

Figure 2. Solutions of the Fisher-KPP equation at integer times with $h=0.1, \delta x=0.1, N=10$ (left) and $h=0.02, \delta x=0.05$ and $N=50$ (right).

We see that the numerical solutions always satisfy $u_{k}^{i} \in[0,1]$ as for the exact solution 1 . Moreover, we can numerically compute that the velocity of the propagating front converges for large times toward $v=2$ as expected (see [2]).

Note that this method is not the same as the particle method proposed by Puckett in 12 and analysed in 1. In particular, in the presentation above, the interpolation grid remains fixed in time, which is a limitation in comparison with particle methods for the approximation of the long time behaviour of the solution in this particular case.

4.3. Ginzburg-Landau equation. We eventually consider the quintic complex Ginzburg-Landau equation in $\mathbb{R}^{2}$ :

$$
\partial_{t} u=m_{0} \Delta u+m_{1} u+m_{2}|u|^{2} u+m_{3}|u|^{4} u
$$

where $u(t, x) \in \mathbb{C}$, and $m_{i} \in \mathbb{C}, i=1,2,3$. This equation exhibits stable pulse-like solution for some values of the parameters (see [16]).

When $m_{0}>0$, we can extend the numerical schemes (4.1)-(4.2) to this case using complex interpolation. We use the parameters $m_{0}=0.2, m_{1}=-0.1, m_{2}=4+i$

\footnotetext{
${ }^{1}$ This can be easily proved from (4.2) for $h$ sufficiently small.
} 
and $m_{3}=-2.75+i$. As the initial solution, we take

$$
u_{0}(x, y)=\exp \left(-\frac{1}{2} x^{2}-\frac{1}{2}|y+q|^{2}+i p y\right)+\exp \left(-\frac{1}{2} x^{2}-\frac{1}{2}|y-q|^{2}-i p y\right)
$$

with $q=1.9$ and $p=0.15$. We use the numerical scheme (4.2) with the Euler scheme $\Phi_{h}$. We take $\delta x=0.05, h=0.01$ and $N=40$. The results are plotted in Figure 3 where we see the collapse of these two pulses. These results can be compared to those in [5].
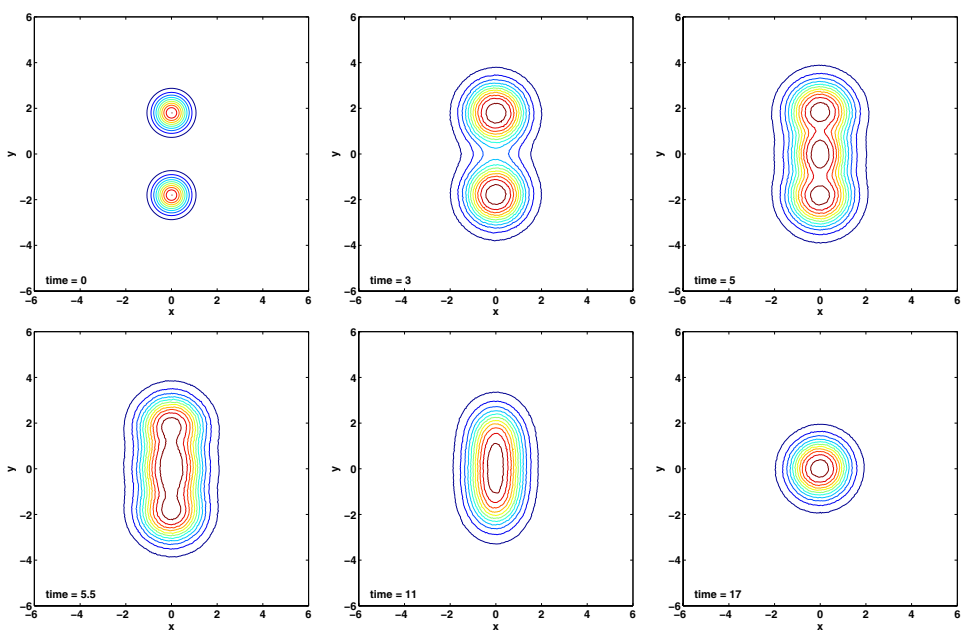

Figure 3. Solutions of Ginzburg-Landau system at the time $t=$ $0,3,5,5.5,11$ and 17 with $\delta x=0.05, h=0.01$ and $N=40$.

\section{ACKNOWLEDGMenT}

The author is glad to thank Arnaud Debussche for his helpful comments and support during the preparation of this work.

\section{REFERENCES}

1. P. Bernard, D. Talay, L. Tubaro, Rate of convergence of a stochastic particle method for the Kolmogorov equation with variable coefficients. Math. Comp. 63 (208) (1994), 555-587. MR.1250770 (95c:65139)

2. E. Brunet and B. Derrida, Microscopic models of traveling wave equations. Comp. Phys. Comm. 121-122 (1999), 376-381.

3. S. Descombes, Convergence of a splitting method of high order for reaction-diffusion systems. Math. Comp. 70 (236) (2001), 1481-1501. MR.1836914 (2002c:65152)

4. S. Descombes and M. Massot, Operator splitting for nonlinear reaction-diffusion systems with an entropic structure: Singular perturbation and order reduction. Numer. Math. 97 (2004), 667-698. MR.2127928 (2006i:65131)

5. S. Descombes and M. Ribot, Convergence of the Peaceman-Rachford approximation for reaction-diffusion systems. Numer. Math. 95 (2003), 503-525. MR2012930(2004i:65076)

6. S. Descombes and M. Schatzman, On Richardson Extrapolation of Strang's Formula for Reaction-Diffusion Equations. in Equations aux Dérivées partielles et Applications, articles dédiés á Jacques-Louis Lions, Gauthier Villars Elsevier, Paris, 1998. MR.1648232 (99i:65006)

7. S. Descombes and M. Schatzman, Strang's formula for holomorphic semi-groups. J. Math. Pures Appl. 81 (2002), 93-114. MR1994884 (2005g:35008)

8. R. A. Fisher, The wave advance of advantageous genes. Annals of Eugenics 7 (1937), 355-369. 
9. T. Ichinose and H. Tamura, Error bound in trace norm for Trotter-Kato product formula of Gibbs semigroups. Asympt. Anal. 17 (4) (1998), 239-266. MR1656823 (2000a:47098)

10. T. Jahnke and C. Lubich, Error bounds for exponential operator splittings. BIT 40 (4) (2000), 735-744. MR:1799313 (2001k:65143)

11. A. Kolmogorov, I. Petrovsky and N. Piscounov, Etude de l'équation de la diffusion avec croissance de la quantité de matière et son application à un problème biologique. Bull. Univ. État Moscou, A 1, (1937), 1-25.

12. E.G. Puckett, Convergence of a random particle method to solutions of the Kolmogorov equation. Math. Comp. 52 (186) (1989), 615-645. MR964006 (90h:65008)

13. M. Schatzman, Numerical integration of reaction-diffusion systems. Numer. Algo. 31 (2002), 247-269. MR1950924 (2004a:65118)

14. D. Talay, Probabilistic numerical methods for partial differential equations: Elements of analysis. Lecture Notes in Math. 1627 (1995), 148-196. MR1431302 (98j:60092)

15. S. Takanobu, On the error estimate of the integral kernel for the Trotter product formula for Schrödinger operator. Annals of Probability 25 (4) (1997), 1895-1952. MR 1487441 (99i:60134)

16. O. Thual and S. Fauve, Localized structures generated by subcritical instabilites. J. Phys. France 49 (1988) 1829-1833.

inRia \& Ecole Normale Supérieure de Cachan Bretagne, Avenue Robert Schumann, 35170 Bruz, France

E-mail address: Erwan.Faou@inria.fr 